\title{
Intra-pulse Cavity Enhanced Measurements of Carbon Monoxide in a Rapid Compression Machine
}

\author{
Ehson F. Nasir, Aamir Farooq \\ King Abdullah University of Science and Technology (KAUST), Clean Combustion Research Center (CCRC), Physical Science and Engineering \\ Division (PSE), Thuwal, 23955-6900, Saudi Arabia \\ Authore-mail address: aamir.farooq@kaust.edu.sa
}

\begin{abstract}
A laser absorption sensor for carbon monoxide concentration was developed for combustion studies in a rapid compression machine using a pulsed quantum cascade laser near $4.89 \mu \mathrm{m}$. Cavity enhancement reduced minimum detection limit down to $2.4 \mathrm{ppm}$ at combustion relevant conditions. Off-axis alignment and rapid intra-pulse down-chirp resulted in effective suppression of cavity noise.

OCIS codes: (280.1740) Combustion diagnostics; (280.3420) Laser sensors; (280.4788) Optical sensing and sensors
\end{abstract}

\section{Introduction}

Rapid compression machines (RCMs) are important tools for investigating ignition characteristics of hydrocarbon fuels at conditions relevant to low-temperature oxidation chemistry (700 - $900 \mathrm{~K}, 10-40$ bar) [1]. RCMs provide a nearly isentropic, homogeneous environment for evaluating chemical kinetic models of low-temperature combustion. Validation of such models requires time-resolved concentration measurements of intermediate species, such as carbon monoxide (CO) [2]. Moreover, to maintain a uniform temperature field during the experiment, dilute reactant gas mixtures must be used to reduce magnitude of heat release, thus increasing sensitivity demands on concentration sensors. In this work, we report the application of a mid-IR laser sensor based on cavity enhanced absorption spectroscopy (CEAS) for CO detection in an RCM. The sensor provides significant improvement in minimum detection limit over single-pass direct absorption.

\section{Sensor Description}

A distributed feedback quantum cascade laser (DFB-QCL), described previously in [3], was used in pulsed mode to probe the $\mathrm{P}(23)$ molecular transition of $\mathrm{CO}$ near $4.89 \mu \mathrm{m}$. The laser was aligned in an off-axis arrangement with a cavity mounted on the RCM combustion chamber. The RCM, details of which can be found in [3], has an inner diameter of $5.08 \mathrm{~cm}$ which serves as the single pass path-length. Two $\mathrm{CaF}_{2}$ concave mirrors, sourced from RMI Co. with a nominal reflectivity of $99.5 \%$, were used to form the cavity. The laser signal was recorded on a $500 \mathrm{MHz}$ bandwidth MCT detector from Vigo System, and acquired at $100 \mathrm{MHz}$ sampling frequency by an oscilloscope.

The QCL was operated at $100 \mathrm{kHz}$ repetition rate with a $500 \mathrm{~ns}$ pulse duration. This provided rapid intra-pulse down-chirp, averaging $6.52 \mathrm{~cm}^{-1} / \mu \mathrm{s}$, as measured using a Germanium etalon. The rapid frequency scan coupled with the off-axis alignment resulted in cavity noise being effectively suppressed, as illustrated by the laser intensity trace in Fig. 1 (right). The single pass absorbance $A_{S P}$ is related to the CEAS absorbance via $A_{\mathrm{CEAS}}=\ln \left(1+G A_{S P}\right)$. In turn, using spectroscopic parameters from HITRAN [4], the species mole fraction $X$ can be inferred from the Beer Lambert relation: $A_{S P}=\left(S P X L \Phi_{v}\right)$. In static cell measurements with known $C O / \mathrm{N}_{2}$ mixtures, the gain factor $G$ was found to be $133 \pm 8$, thus the measured mirror reflectivity is $99.25 \pm 0.04 \%$.
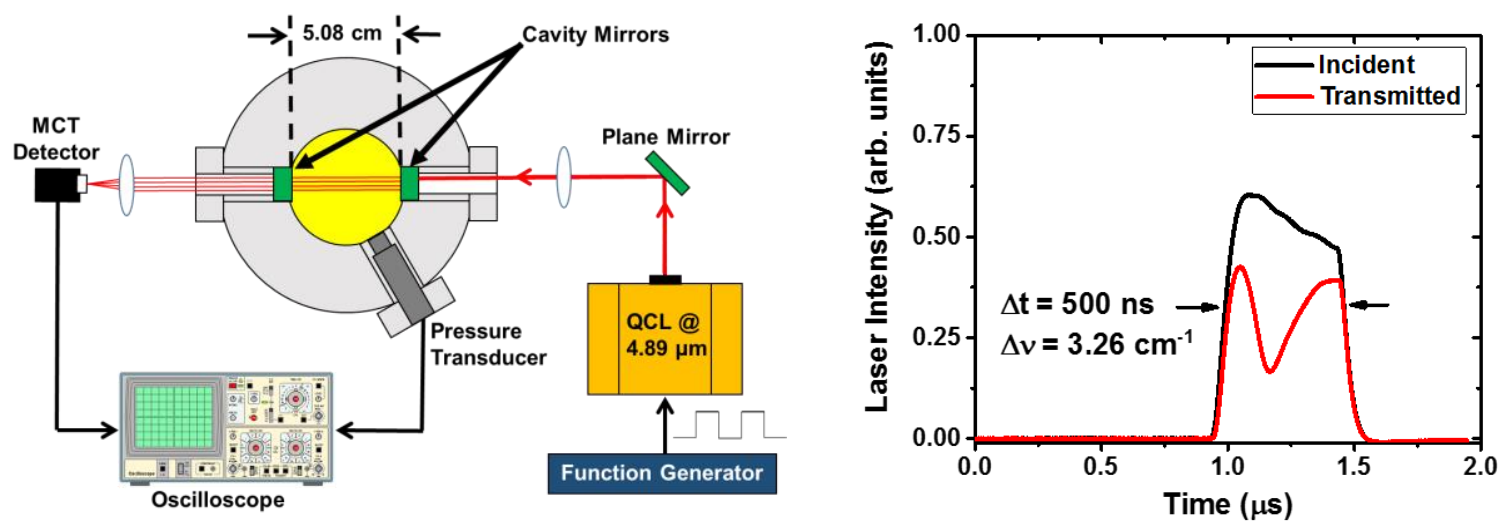

Fig. 1. Left: Optical Schematic of CEAS sensor aligned on the cross-section of the RCM combustion chamber. Right: Laser intensity trace for static cell measurements at $80^{\circ} \mathrm{C}, 7$ bar. 


\section{Experimental Results}

Figure 2 (left) shows simulated comparison of detection limits between CEAS and single-pass arrangements for the $\mathrm{P}(23) \mathrm{CO}$ line at typical RCM conditions. At $800 \mathrm{~K}$, the CEAS technique produces a reduction in the minimum detection limit by factor of 63 (2.4 ppm down from $151 \mathrm{ppm})$.
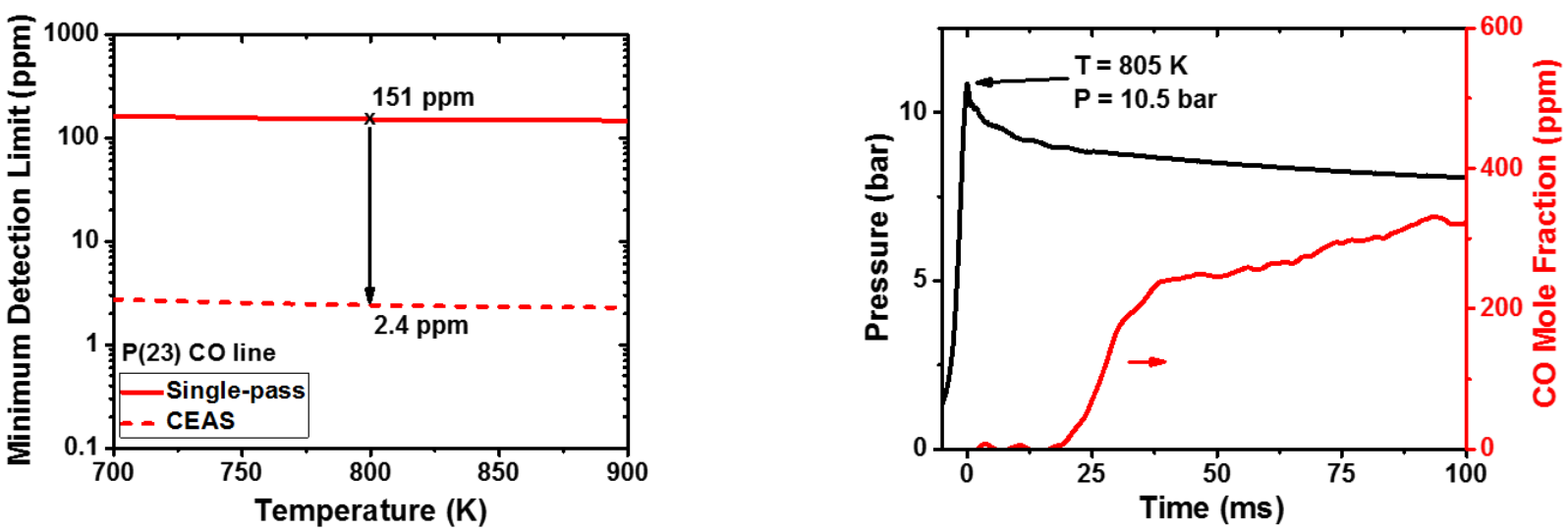

Fig. 2. Left: Minimum detection limit of $\mathrm{CO}$ using $\mathrm{P}(23)$ absorption line. Right: Measured CO concentration and pressure profile for the oxidation of $0.2 \% n$-octane / $2.5 \%$ oxygen / $97 \%$ nitrogen.

The sensor was used for measuring CO species time-histories for $n$-octane oxidation as shown in Fig 2 (right). The measurements have an effective time resolution of $10 \mu \mathrm{s}$ over a total measurement time window of $100 \mathrm{~ms}$, the typical residence time for most RCM facilities. High frequency noise from pulse-to-pulse variations as well as sampling bit noise from the oscilloscope was removed from the $\mathrm{CO}$ profile using a $2 \mathrm{kHz}$ low-pass filter in postprocessing. Time zero for the pressure trace is set to the end-of-compression phase, which in this case occurs at the maximum point of the measured pressure trace. The onset of $\mathrm{CO}$ formation at about $25 \mathrm{~ms}$ corresponds to the lowtemperature first-stage ignition. For stoichiometric $n$-octane/air mixtures (i.e., $\varphi=1$ ), $1.65 \%$ mole fraction of $n$-octane is required in the gas mixture. Here however, only $0.2 \% n$-octane was used, significantly reducing reactivity and suppressing heat release, as is evident from the pressure trace in Fig. 2 (right). Such measurements in dilute mixtures are highly useful for validation the low-temperature reaction kinetics.

\section{Conclusions}

A laser sensor based on the CEAS technique was demonstrated for CO measurements in an RCM. The sensor provided high time resolution $(10 \mu \mathrm{s})$ as well as more than an order of magnitude higher sensitivity than a conventional single-pass absorption technique. The decreased detection limit allows for dilute reactive mixtures to be used for validating kinetic models, ensuring a reactive system free from transport effects.

\section{Acknowledgements}

Research reported in this publication was supported by funding from the King Abdullah University of Science and Technology (KAUST).

\section{References}

[1] C. J. Sung and H. J. Curran, "Using rapid compression machines for chemical kinetics studies," Prog. Energy Combust. Sci. 44, 1-18 (2014).

[2] A. Tekawade, G. Kosiba, and M. A. Oehlschlaeger, "Time-resolved carbon monoxide measurements during the low- to intermediatetemperature oxidation of n-heptane, n-decane, and n-dodecane," Combust. Flame 173, 402-410 (2016).

[3] E. F. Nasir and A. Farooq, "Time-resolved temperature measurements in a rapid compression machine using quantum cascade laser absorption in the intrapulse mode," Proc. Combust. Inst. 36, 4453-4460 (2017).

[4] I. E. Gordon, L. S. Rothman, C. Hill, et al., "The HITRAN2016 molecular spectroscopic database," J. Quant. Spectrosc. Radiat. Transfer 203, 3-69 (2017). 